\title{
A "sneaky" symptom of aortic dissection. Brief literature review, physiopathology and diagnostic tools management
}

\author{
Carlo Uran', Angela Giojelli2 \\ ${ }^{1}$ Cardiology and Intensive Care Unit, "San Giuseppe e Melorio" Hospital, Santa Maria Capua Vetere (CE); \\ ${ }^{2}$ Diagnostic Imaging Unit, "San Giuseppe e Melorio" Hospital, Santa Maria Capua Vetere (CE), Italy
}

\begin{abstract}
Aortic diseases cover a large spectrum of conditions, such as aortic aneurysm and acute aortic syndromes (i.e., dissections, intramural hematoma, penetrating atherosclerotic ulcer, traumatic aortic injuries, and pseudoaneurysms), genetic diseases (e.g., Marfan syndrome) and congenital abnormalities, such as coarctation of the aorta. These conditions may have an acute presentation; thus, if the acute aortic syndrome is the first sign of the disease, the prognosis is extremely poor. Prompt diagnosis and timely therapy are therefore mandatory. In this paper, we discuss a deceptive symptom of painless aortic dissection and its physiopathology. Furthermore, we briefly review the literature and discuss the management of diagnostic tools.
\end{abstract}

Correspondence: Dr. Carlo Uran, Cardiology and Intensive Care Unit, "San Giuseppe e Melorio" Hospital, Via Melorio, 81055 Santa Maria Capua Vetere (CE), Italy.

Mobile: +39.3392683713

E-mail: carlura@libero.it

Contributions: All the authors made a substantive intellectual contribution, have read and approved the final version of the manuscript and agreed to be accountable for all aspects of the work.

Ethics approval: All procedures performed in connection with this case report were in accordance with the ethical standards of the institutional or national research committee and with the 1964 Helsinki Declaration and its later amendments.

Conflict of interest: The authors declare that they have no competing interests, and all authors confirm accuracy.

Key words: Aortic dissection; headache; echocardiogram; transesophageal echocardiogram; CT angiography; MRI.

Received for publication: 8 September 2020.

Accepted for publication: 28 December 2020.

${ }^{\circ}$ Copyright: the Author(s), 2021

Licensee PAGEPress, Italy

Monaldi Archives for Chest Disease 2021; 91:1662

doi: 10.4081/monaldi.2021.1662

This article is distributed under the terms of the Creative Commons Attribution Noncommercial License (by-nc 4.0) which permits any noncommercial use, distribution, and reproduction in any medium, provided the original author(s) and source are credited.

\section{Introduction}

Aortic dissection (AD) is a severe disease with a high mortality rate. Unfortunately, it may be diagnosed late because of the high frequency of unusual symptoms at the onset. The onset of $\mathrm{AD}$ is typically characterized by chest or abdominal pain, or sometimes by back pain. The literature reports several cases with atypical, painless onset, which makes the diagnosis extremely difficult.

\section{Case Report}

A 58-year-old man was taken by ambulance to the emergency department (ED) of our hospital. He was unconsciousness and had hyperextension and internal rotation of the upper and lower limbs. Owing to the man's clinical condition, the anamnesis was taken by speaking with his relatives, who reported that he smoked about 20 cigarettes/day, was on therapy with antihypertensive drugs and, in the last seven days, had suffered from headache, for which he had taken anti-inflammatory drugs, without any benefit. His relatives reported that he had suddenly passed out at home 40 min earlier. No chest, neck or back pain was referred. Furthermore, his relatives reported that he had an 'enlarged aorta'. His family history did not reveal any genetic disease or notable clinical events.

Owing to the man's clinical condition, a consultant cardiologist and resuscitator were called. Blood pressure measured in the right arm was $120 / 60 \mathrm{mmHg}$ but was undetectable in the left arm. Moreover, both right radial and femoral pulses were detectable, while the left ones were not. A peripheral vein was cannulated and a blood sample was taken for urgent chemistry tests; an arterial blood gas test was also performed. Neither of these showed any noteworthy alterations. EKG showed sinus bradycardia (54 bpm). In order to protect the airways and the central nervous system, the patient underwent orotracheal intubation and mechanical pulmonary ventilation. Cardiac auscultation revealed a loud grade3 (Levine scale) systolic murmur. The echocardiogram showed normal left ventricular ejection fraction (55\%) and dilation of the ascending aorta with evidence of a dissection flap (Figure 1A). No pericardial effusion was present. Ultrasound Doppler of the supraaortic trunks revealed a severe reduction of blood flow through the common carotid arteries. These findings aroused the diagnostic suspicion of $\mathrm{AD}$; the patient therefore underwent CT angiography, in order to confirm the AD and to assess its extension, and a head CT in order to evaluate any brain damage. The head CT showed ischemia of the brain stem (Figure 1B). The CT angiography revealed an aneurysm of the ascending aorta $(52 \mathrm{~mm})$ and confirmed the presence of a type-A AD; this originated from the 
supravalvular plane (Figure 1C), extended to the arch (Figure 1D) and involved the brachiocephalic trunk, common carotid arteries and left subclavian artery (Figure 2 A,B). Dissection of the descending aorta, occlusion of the superior mesenteric artery, narrowing of the right renal artery, due to its probable involvement in the dissection (Figure 2C), and occlusion of the right iliac arterial axis (Figure 2D) were also detected.

Because of these findings, the patient was urgently transferred to the hub hospital for cardiac surgery. A few minutes after arrival, however, his clinical condition worsened: atrioventricular dissociation appeared on the EKG monitor, followed by an idioventricular rhythm and subsequent cardiac arrest. Cardiopulmonary resuscitation was attempted, but the patient died.

\section{Literature review}

The incidence of acute AD is about 2-3.5 cases per 100,000 people per year. A 2010 study by the Global Burden of Disease project showed an increase in deaths due to ascending aortic aneurysm, from 2.49 to $2.28 / 100,000$, with a higher incidence among males. By contrast, the prevalence and incidence of abdominal aortic aneurysm were seen to have decreased [1]. The main classifications of AD were drawn up by DeBakey and by Stanford (Table 1). This latter is useful in assessing the optimal management of this dramatic event, as type-A dissection needs emergency surgery. Like many vascular diseases, aortic disease may be diagnosed late, as it may remain subclinical for variable periods of time; its prognosis is therefore often poor.

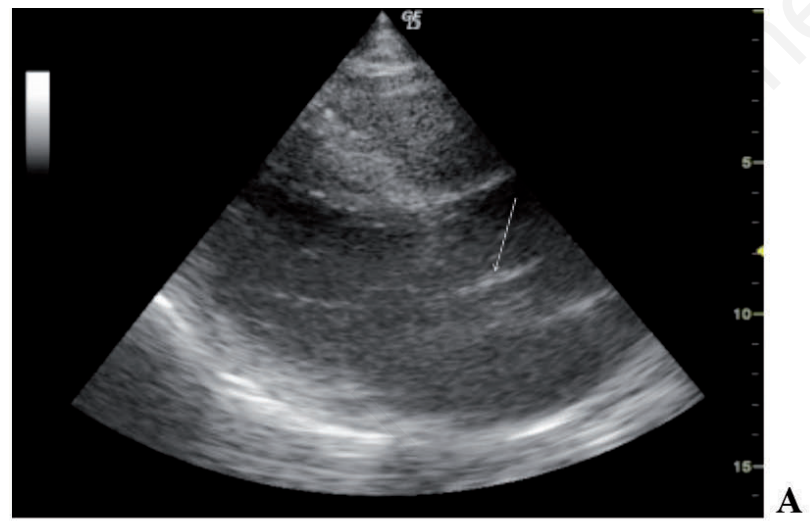

A

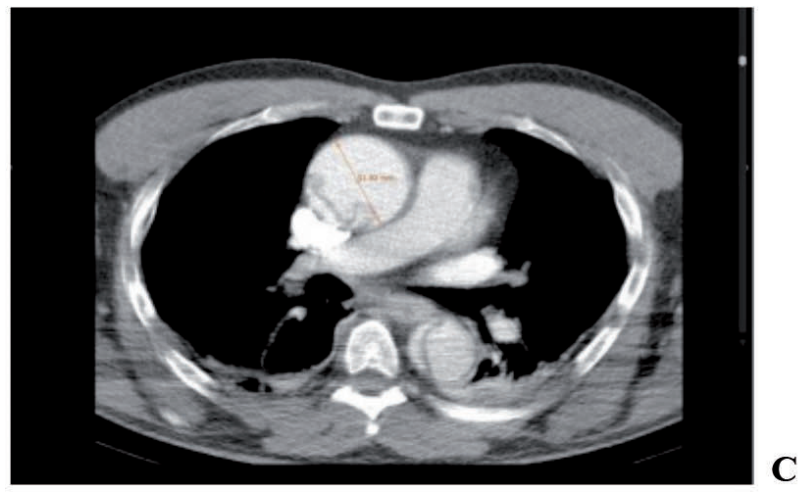

C
Aortic diseases comprise many conditions: aortic aneurysms, acute aortic syndromes, including $\mathrm{AD}$, intramural hematoma, penetrating atherosclerotic ulcer and traumatic aortic injury, pseudoaneurysm, aortic rupture, atherosclerotic and inflammatory affections, genetic diseases (e.g. Marfan syndrome) and congenital abnormalities (e.g. coarctation of the aorta). Acute AD is a dramatic condition with a high mortality rate [3]. Patients with a type-A AD present severe chest pain (78\%), less frequently abdominal pain, syncope and ischemic neuropathy. Isolated headache and neck pain as onset symptoms are very infrequent, as involvement of the proximal common carotid artery is somewhat rare. Indeed, few cases of AD presenting with headache are described in the literature. Singh et al. reported a case of a patient presenting bifrontal headache who became hemodynamically unstable. CT angiography revealed a Stanford type-A AD [3]. Ko and Park also reported a case of a patient who presented bifrontal headache and, on further evaluation, was diagnosed with common carotid artery dissection together with AD [4]. Parikh reported a case of a 71-year-old Caucasian male with a medical history of choroideremia, who presented to the ED because of a left-sided temporoparietal headache upon waking, but who had no focal neurological deficits on the day of admission. Contrast $\mathrm{CT}$ of the chest revealed a thoracic $\mathrm{AD}$ involving both the ascending and descending aorta, with an intramural hematoma in the descending aorta. The dissection extended superiorly to the brachiocephalic and left common carotid trunks [5].

Notably, headache may be present in patients with primary carotid artery dissection [6]. By contrast, transient or permanent neurological symptoms at the onset of $\mathrm{AD}$ are frequent (17-40\% of patients); these are often severe and may mask the underlying condition. In the case of pain-free dissection (which occurs in 5-

Figure 1. A) Ascending aortic aneurysm and dissection flap (arrow). B) Ischemia of brain stem (arrow). C) Ascending aortic dissection and diameter. D) Aortic arch dissection. 
$15 \%$ of cases) with predominant neurological symptoms, the diagnosis of AD may be difficult and delayed [7]. A study published in 2007 by Gaul et al. showed that only two thirds of patients with neurological symptoms at the onset referred pain. By contrast, most patients without neurological symptoms experienced pain (94\%) [8]. In a retrospective study, Bossone et al. showed that patients suffering from stroke due to type-A AD presented chest and back pain less frequently than those without stroke $(69.7 \%$ vs $82.3 \%$, $32.4 \%$ vs.43.4\%, respectively) [9]. Whereas the study by Gaul et al. included in the symptomatic group all neurological symptoms, such as peripheral nerve deficits, Bossone et al. included only stroke [9]. A study published in 2017 by Shono et al. showed that $18.6 \%$ of type-A AD patients had central nervous system symptoms at the onset, and all of these had disturbances of consciousness, stroke and ischemic encephalopathy [10].

The timing of surgery in ascending aortic aneurysm (AsAA) is a matter of debate. In the risk assessment of dissection or rupture, the annual growth rate is relevant. Notably, the factors associated with annual growth rate not only vary significantly but are often contradictory between studies. In 2017, Park reported that baseline aortic diameter correlated positively with increased growth rate [11]. By contrast, another study found that baseline aortic diameter was not correlated with the aortic dilatation rate [12]. One study even reported a correlation between higher annual aortic growth rate and lower baseline aortic diameter. Renal failure, female sex, aortic stenosis and aortic regurgitation were also identified as being

Table 1. Classifications of aortic dissection.

\author{
DeBakey classification of acute aortic syndromes \\ Type I involves the ascending aorta, the aortic arch and the thoracic aorta \\ Type II limited to the ascending aorta \\ Type III involves the descending aorta distally to the origin of the left subclavian artery \\ - Type III-A limited to thoracic aorta \\ - Type III-B involves thoracic and abdominal aorta
}

Stanford classification of acute aortic syndromes

Type A involves the ascending aorta and can extend towards the descending thoracic aorta up to the iliac arteries

Type B involves the descending aorta distally to the origin of the left subclavian artery, with or without distal extension
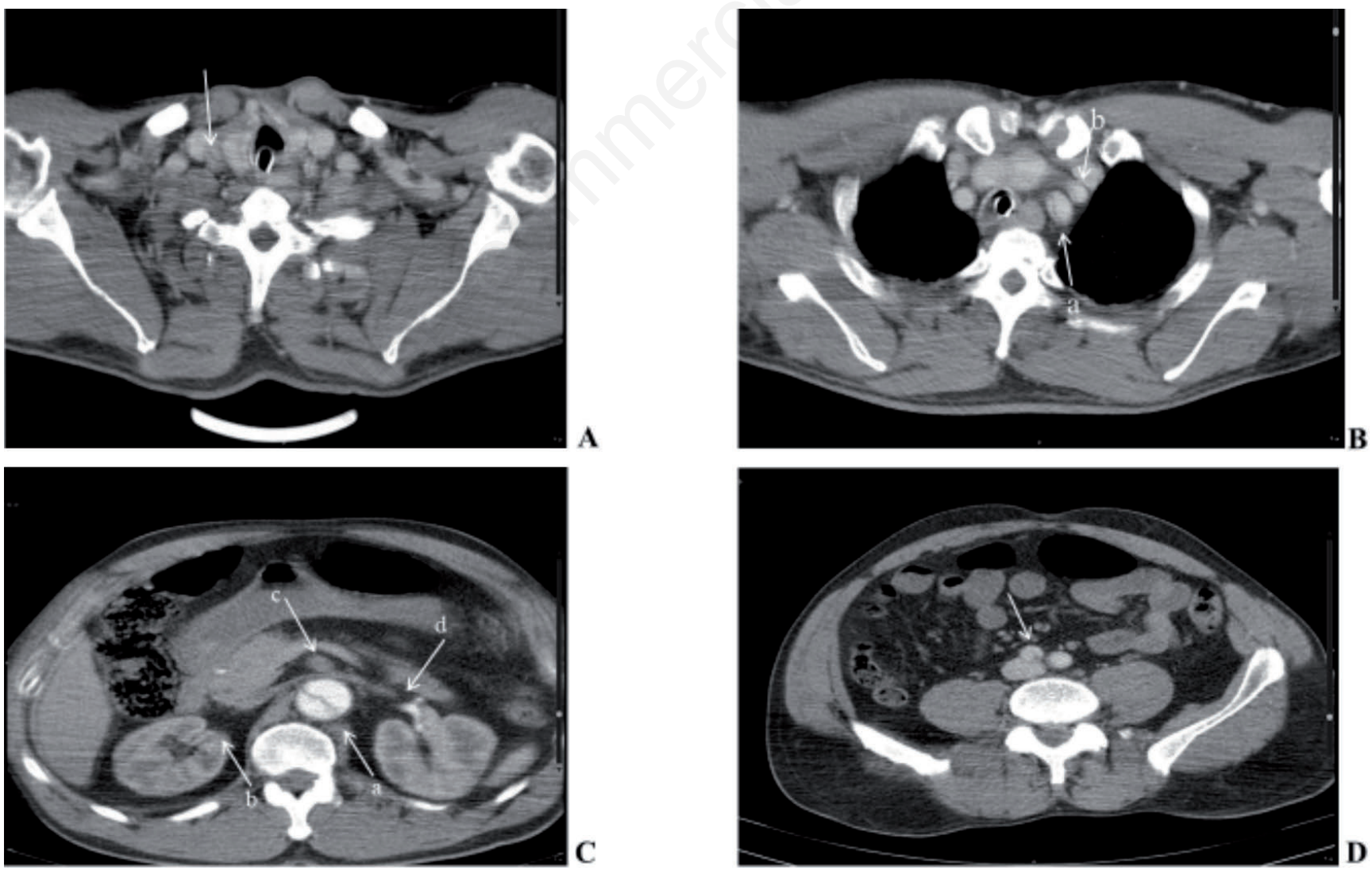

Figure 2. A) Right common carotid artery dissection. B) Left subclavian artery dissection (arrow a); left common carotid artery dissection (arrow b). C) Abdominal aortic dissection (arrow a); right renal artery narrowed (arrow b); superior mesenteric artery occluded (arrow c); normal right renal artery (arrow d). D) Right common iliac artery dissection. 
associated with higher growth rate [13]. The use of anticoagulants and statins has been reported to be related with a lower growth rate [14]. However, it is noteworthy that the most important factor related with an increased risk of dissection and rupture is aneurysm size [15].

\section{Discussion}

\section{Physiopathology: Why headache?}

Several hypotheses have been put forward to explain how AD can induce headache. Distension of blood vessels in the neck or ischemia of the peri-carotid plexus may play a role [16]. It is noteworthy that headache in $\mathrm{AD}$ might originate from ischemia of the aortic wall itself. Indeed, arterial walls are concomitantly nourished both by the vasa vasorum and by diffusion from the lumen of the vessel. Diffusion is more important in small blood vessels, in which vasa vasorum are sometimes absent. In larger blood vessels with a thicker wall, however, diffusion alone is not enough to ensure an adequate supply of oxygen and nutrients to the arterial wall, which is why the vasa vasorum become indispensable [17]. Consequently, dissection may lead to arterial wall ischemia by means of several processes: i) interruption of the blood flow in the vasa vasorum due to thrombosis of the false lumen or stenosis of the true one; ii) thrombosis of the vasa vasorum itself; iii) compression of the vasa vasorum due to expansion of the hematoma in the false lumen and, finally, iv) stress- and ischemia-induced reflex vasoconstriction of the vasa vasorum due to sympathetic activation [18]. Ischemia of the aortic wall leads to a pathological activation of the trigeminal vascular system and causes headache. This happens through two different pathways: humoral and anatomical. The humoral pathway requires that inflammatory mediators formed in the ischemic aortic wall be released into the bloodstream and reach the central nervous system, where they can directly stimulate specific receptors. Serotonin is considered the most important of these inflammatory mediators and acts on 5hydroxytryptamine $1 \mathrm{D}$ receptors on trigeminal perivascular projections or within the trigeminal nucleus caudalis [19]. Several other pain-related mediators have been described, notably prostaglandins, bradykinin, adenosine triphosphate, histamine, nitric oxide, potassium and hydrogen ions [20].

The anatomical pathway implies that pain signals generated by nociceptors of the aortic wall are transferred to the trigeminal ganglion via the cardiac plexus. This plexus is very peculiar because the small nerve branches which form it originate from cervical sympathetic ganglia rather than from thoracic ganglia. On the other hand, there is a direct connection between the first cervical ganglion and the trigeminal ganglion through the internal carotid nerve [21]. Thus, pain signals generated by nociceptors in the aortic wall suffering ischemia could be transmitted to the trigeminal ganglion and then to the trigeminal nucleus caudalis in the lower brainstem and upper cervical cord. This information would be further relayed to the contralateral ventral posteromedial thalamic nuclei via the trigemino-thalamic tract and finally to somatosensory cortices [22].

\section{Management of diagnostic tools}

As mentioned above, headache is an extremely rare onset symptom of AD. It is noteworthy that headache may be present in several conditions, such as trigeminal neuralgia, cerebral vascular malformations (cavernous angioma, cerebral aneurysm), vertebral artery dissection, carotid artery dissection, venous cerebral thrombosis, subdural hematoma, and intracranial hypertension, as well as in ascending AD. Every one of these conditions has to be considered if a patient reports headache, and an echocardiogram should be performed in order to evaluate the ascending aorta, especially in patients who refer 'enlarged aorta'; indeed, in the event of dissection, mortality is extremely high, especially if surgery is not undertaken, ranging from 1 to $2 \%$ per hour and reaching about $26 \%$ in total [23]. The most common cause of death is aortic rupture, followed by stroke, visceral ischemia, cardiac tamponade and cardiovascular failure. In the case of our patient, headache may have been misinterpreted by the patient himself as paroxysmal migraine; however, as discussed above, it was very probably the onset symptom of the AD. It is noteworthy that headache may be the only sign of dissection, and often precedes the onset of ischemic signs; moreover, the time between the headache and the stroke is variable, ranging from a few seconds to weeks (as in our patient) [24]. It therefore requires prompt diagnosis and treatment. In cases of unexplained headache or neck pain in young patients, it is crucial to suspect an arterial dissection, in order avoid cerebrovascular events. The clinical suspicion must first be investigated by means of ultrasound of the aorta and the supra-aortic vessels, and subsequently, if any doubt remains, by an appropriate imaging study.

Here, some considerations need to be made. Firstly, in patients suffering from headache, all the possible diagnoses listed above must be taken into account. Secondly, in all patients with cardiovascular risk factors who arrive at the ED suffering from headache that is unresponsive to common anti-inflammatory drugs, ultrasound evaluation of the ascending aorta and supra-aortic trunks should be performed. Finally, since a cardiologist might not always be available, the ED physicians should be able to perform transthoracic echocardiography (TTE) in order to promptly evaluate the ascending aorta in patients suspected of having a type-A AD. TTE can be used as a rapid first-line tool to triage patients with suspected type-A AD; however, it cannot be used as a stand-alone test to rule in/out type-A AD. TTE can rapidly provide crucial diagnostic information in patients presenting with shock or hypotension. It is recommended for an initial evaluation, with class I indication and evidence level C. Finally, transthoracic ultrasound has a fundamental role in type-A AD, in order to rule out pericardial tamponade, contained rupture and severe aortic insufficiency $[25,26]$. Consequently, it would be extremely useful for noncardiologists to undergo training in cardiac ultrasound.

A brief research report published by Scott et al. in 2015 showed that dissection was extremely rare, which is why it is often misdiagnosed [27]. Moreover, a 2010 study found that only $71 \%$ of patients with type-A dissection had chest pain, and $6 \%$ had no pain altogether [28]. In addition, AD may present with symptoms such as heart failure, neurological deficits, syncope, or vascular insufficiency, which are more commonly found in other diseases. For these reasons, AD may be misdiagnosed in $16 \%$ of cases presenting to the ED [29]. However, this percentage is certainly underestimated, as patients with dissection, especially those who die, may not have a proper diagnosis. In this regard, clinical history and physical examination may not be very reliable, in that physicians correctly suspect $\mathrm{AD}$ after the initial clinical evaluation in only $65 \%$ of patients [30]. Indeed, while the sudden onset of severe pain accompanied by high blood pressure and pulse deficits suggests dissection, the absence of these clinical signs does not exclude it at all. Chest x-ray can be used as a screening tool, but signs such as abnormal aortic contour and enlarged mediastinum have sensitivity values ranging from $9 \%$ to $71 \%$ [31]. It is therefore clear that none of the above approaches alone is sufficient to diagnose AD. Unfortunately, failure or delay in diagnosing AD may lead to significant mortality. 
CT angiography is the most reliable diagnostic tool (Class I indication, evidence level $\mathrm{C}$ ) and it is mandatory that patients undergo this examination whenever there is a suspicion of $\mathrm{AD}$, as reported in the ESC Guidelines on the diagnosis and treatment of aortic diseases (2014) and in the position statement by the European Association for Cardio-Thoracic surgery and the European Society for Vascular Surgery (2019). The Class I, evidence level C indication stems because CT angiography is the most reliable imaging technique for the diagnosis of $\mathrm{AD}$, because of its speed, widespread availability and excellent sensitivity of $95 \%$ in AD. Moreover, its sensitivity and specificity in diagnosing arch vessel involvement are $93 \%$ and $98 \%$, respectively, with an overall accuracy of $96 \%$. Diagnostic findings include active contrast extravasation or high-attenuation hemorrhagic collections in the pleura, pericardium, or mediastinum. The multi-planar reconstruction images play a fundamental role in confirming the diagnosis and in determining the extent of dissection. Indeed, multidetector $\mathrm{CT}$ can precisely measure the extent of dissection, the length and diameter of the aorta, the true and the false lumen and the involvement of vital vasculature.

A new tool is 'Triple-rule-out' CT, which is an ECG-gated, 64detector CT study used in the ED to evaluate patients with acute chest pain with three potential causes: AD, pulmonary embolism, and coronary artery disease. The advantage of Triple-rule-out CT is that it rapidly investigates life-threatening sources of acute chest pain and has a high negative predictive value. However, it is important to recognize any highly mobile linear intraluminal filling defect, which may mimic an intimal flap on CT. A 'pulsation artefact' is the most common cause of misdiagnosis; this is caused by pulsatile movement of the ascending aorta during the cardiac cycle between end-diastole and end-systole. For this reason, the simple CT angiography approach is outdated. Indeed, CT angiography must be performed with ECG gating in order to suppress motion artifacts, or else by means of a $180^{\circ}$ linear interpolation reconstruction algorithm. Dense contrast enhancement in the left brachiocephalic vein or superior vena cava, mediastinal clips, and indwelling catheters can all produce streak artefacts in the aorta, which may potentially simulate dissection. This difficulty can be avoided by paying careful attention to the volume and injection rate of intravenous the contrast material administered [25-32].

Trans-esophageal echocardiography (TEE) also deserves to be mentioned. In 1995, Nicosia et al. [33] designed a study in order to assess the sensitivity, specificity and diagnostic accuracy of TEE and X-ray contrast-enhanced $\mathrm{CT}$ in the diagnosis of $\mathrm{AD}$ and its complications. The study showed that the sensitivity of TEE in detecting type-A AD was $100 \%$, a significantly higher $(\mathrm{p}<0.05)$ value than that of CT $(77.2 \%)$. However, the two imaging procedures did not differ significantly in the detection of type-B dissection (both have a $>87 \%$ of sensitivity). The specificity of TEE in the detection of type-A AD was $94 \%$, which was not significantly higher than that of CT $(86.6 \%)$. Neither TEE nor CT yielded false negative results in the diagnosis of type-B AD. TEE proved reliable in correctly identifying the primary entry site in the ascending aorta $(80 \%)$, the arch $(62.5 \%)$ and descending aorta $(71.4 \%)$, and also in identifying the involvement of coronary arteries $(62.5 \%)$ and aortic arch branch vessels (71.4\%). Aortic regurgitation was accurately identified by TEE in each case. Both TEE and CT correctly identified thrombosis of the false lumen and pericardial effusion. In all patients, intraoperative TEE documented post-repair persistence of the intimal flap. The study concluded that TEE was safe and accurate in diagnosing and classifying AD. Moreover, TEE also provides the diagnostic information necessary for therapeutical decision-making. Finally, intraoperative TEE enables the preoperative diagnosis to be improved and yields important information for the management of the patient during follow-up [2633]. ED physicians should be able to perform TTE in the case of suspected dissection involving the ascending aorta, while leaving the execution of TEE to the cardiologist.

We should also mention magnetic resonance imaging (MRI), which is an accurate tool in the diagnosis of aortic dissection (sensitivity and specificity, 98\%). Magnetic resonance angiography (MRA) is the most commonly used MRI technique for both preand post-procedural imaging of the thoracic aorta. Contrastenhanced MRA techniques rely on the T1-shortening effect of gadolinium-chelate contrast agents in blood to generate a high intravascular signal instead of exploiting the inherent motion of blood flow, as in the flow-based time-of-flight and phase-contrast techniques. Because of this different approach, the vascular signal generated by contrast-enhanced MRA is not hampered by the numerous flow-related artefacts that can degrade the flow-based MRA techniques [34].

Although MRI produces high-resolution 3-D images, it is very rarely performed in critical care facilities, as it is unavailable in most EDs and requires long image-acquisition times. The use of phasedarray coils provides the additional benefit of markedly shortening image-acquisition times or, through the use of parallel imaging schemes, of acquiring image sets of higher spatial resolution in the same time period. As with CT angiography, the vascular enhancement is a transient and dynamic process; hence, the critical element in contrast-enhanced MRA is the proper timing of image acquisition.

Dynamic MRA provides temporal information during the heart cycle, which can be visualized as a dynamic display, thereby adding a fourth dimension: 4-dimensional contrast-enhanced MRA. Its acquisition is typically combined with a gadolinium-based contrast medium injection, while a sequence of $3 \mathrm{D}$ volumes, including fat suppression, is acquired over time. Rapid gradient-echo sequence covers the entire aorta, allowing high temporal resolution of, for example, 2-4 s/volume and an interpolated spatial resolution of 1 $\mathrm{mm}^{3}$ at a static magnetic field strength of 3 Tesla. Cranial MRI can be used in addition to intracranial Doppler ultrasound to assess completeness of the circle of Willis, which helps predict the risk of insufficient cross-flow and stroke. Time-resolved MRA of the thoracic aorta is the optimal method of studying the mobility, stiffness and dynamics of dissection membranes and the resulting static or dynamic large-vessel occlusion mechanisms. Like intracardiac flow dynamics in valvular disease, true and false lumen, antegrade, retrograde and turbulent flows in aortic dissection, should be imaged by means of MRA as the 'gold standard'. MRI cannot be performed in patients with claustrophobia, or in those with not MRI compatible pacemakers or other metal devices. It can be used in patients who are allergic to the iodinated contrast agents used in CT or when CT is inadequate or the true diagnosis remains uncertain. MRI is rarely performed in patients with ascending aortic rupture, as it is difficult to monitor unstable hemodynamic patients during imaging. However, intra-aortic conditions, including mural thrombi, are clearly visualized by MRI, and this information may also help to determine the treatment strategy [35].

\section{Conclusions}

Type- Type-A AD is a severe clinical emergency, the onset of which is sometimes misdiagnosed. Headache is a very deceptive symptom and, albeit extremely rare as the onset symptom of $\mathrm{AD}$, 
should not be overlooked. Sudden painless onset with neurological symptoms is by no means rare and has been observed in one third of cases of dissection reported in the literature. For this reason, the diagnostic pathway must include a thorough anamnesis, as no symptom can be disregarded.

As reported in the ESC Guidelines on the diagnosis and treatment of aortic diseases (2014) and in the position statement of the European Association for Cardio-Thoracic surgery and the European Society for Vascular Surgery (2019), CT angiography is the most reliable diagnostic tool. It is therefore the investigation of choice and is mandatory in the case of suspected aortic dissection. This technique acquires high spatial resolution 3-dimensional images of the aorta and surrounding structures, enables the diagnosis to be made and aids treatment planning. However, simple CT angiography is now outdated, and the examination must be performed with ECG triggering/gating in order to suppress motion artifacts.

MRI is an accurate means of diagnosing acute aortic syndrome; however, it is rarely performed in emergency settings, as it is unavailable in most EDs and requires long image-acquisition times. The use of phased-array coils markedly shortens image-acquisition times and, through the use of parallel imaging schemes, enables image sets of higher spatial resolution to be acquired in the same time period. MRI can be used in patients who are allergic to the iodinated contrast agents used in CT or when CT is inadequate, or the true diagnosis remains uncertain.

Ultrasound (TTE and, mostly, TEE) plays some role in the diagnosis of type-A AAD and every ED physician should be able to perform TTE, which has a smaller field of view than CT and MRI. TTE is a rapid first-line diagnostic tool and can provide functional information with high temporal resolution.

\section{References}

1. Sampson UK, Norman PE, Fowkes FG, et al. Global and regional burden of aortic dissection and aneurysms: mortality trends in 21 world regions, 1990 to 2010. Glob Heart 2014;9:171-80.

2. Erbel R, Aboyans V, Boileau C, et al. 2014 ESC Guidelines on the diagnosis and treatment of aortic diseases: document covering acute and chronic aortic diseases of the thoracic and abdominal aorta of the adult. Eur Heart J 2014;35:2873-926.

3. Singh S, Huang JY, Sin K, Charles RA. Headache: an unusual presentation of aortic dissection. Eur J Emerg Med 2007;14:47-9.

4. Ko J-I, Park T. Headache: a rare manifestation of Debakey type I aortic dissection. Am J Emerg Med 2014;32;291.e5-6.

5. Parikh M, Agrawal A, Thyagarajan B, et al. Temporoparietal headache as the initial presenting symptom of a massive aortic dissection. Case Rep Emerg Med 2015;2015:626825.

6. Sturzenegger M. Spontaneous internal carotid artery dissection: early diagnosis and management in 44 patients. J Neurol1995;242:231-8.

7. Hagan PG, Nienaber CA,; Isselbacher EM, et al. The international registry of acute aortic dissecion (IRAD). JAMA 2000;283:897-903.

8. Gaul C, Dietrich W, Friedrich I, et al. Neurological symptoms in type A aortic dissections. Stroke 2007;38:292-7.

9. Bossone E, Corteville DC, Harris KM, et al. Stroke and outcomes in patients with acute type A aortic dissection. Circulation 2013;128:S175-9.

10. Shono Y, Akahoshi T, Mezuki S, et al. Clinical characteristics of type A acute aortic dissection with symptom of the central nervous system. Am J Emerg Med 2017;35:1836-8.

11. Park K-H, Chung S, Kim DJ, et al. Natural history of moderately dilated tubular ascending aorta: implications for determining the optimal imaging interval. Eur J Cardiothorac Surg 2017;51:959-64.

12. Gagné-Loranger M, Dumont É, Voisine P, et al. Natural history of 40-50 $\mathrm{mm}$ root/ ascending aortic aneurysms in the current era of dedicated thoracic aortic clinics. Eur J Cardiothorac Surg 2016;50:562-6.

13. Kim JB, Spotnitz M, Lindsay ME, et al. Risk of aortic dissection in the moderately dilated ascending aorta. J Am Coll Cardiol 2016;68:1209-19.

14. Angeloni E, Vitaterna A, Pirelli M, Refice S. Effects of statin therapy on ascending aorta aneurysms growth: a propensitymatched analysis. Int J Cardiol 2015;191:52-5.

15. Vapnik JS, Kim JB, Isselbacher EM, et al. Characteristics and outcomes of ascending versus descending thoracic aortic aneurysms. Am J Cardiol 2016;117:1683-90.

16. Rust H, Kimmig H. Thunderclap headache as the main symptom of an acute aortic dissection type A. J Neurol 2013;260:1925-6.

17. Heistad DD, Marcus ML. Role of vasa vasorum in nourishment of the aorta. Blood Vessels 1979;16:225-38.

18. Tatuene JK, Excoffier S, Vallee JP, Kleinschmidt A. Putative pathophysiological mechanisms in recurrent hemicrania from aortic dissection: a case report. BMC Res Notes 2015;8:246.

19. Ahn AH, Basbaum AI. Tissue injury regulates serotonin 1D receptor expression: implications for the control of migraine and inflammatory pain. J Neurosci 2006;26:8332-8.

20. Dray A. Inflammatory mediators of pain. $\mathrm{Br} \mathrm{J}$ Anaesth 1995; 75:125-31.

21. Rouvière H, Delmas A. [Nerfs du tronc].[In French]. In: Delmas $\mathrm{V}$, editor. Anatomie humaine descriptive, topographique et fonctionnelle. Paris: Masson; 2002. p. 271-98.

22. Almeida TF, Roizenblatt S, Tufik S. Afferent pain pathways: a neuro anatomical review. Brain Res 2004;1000:40-56.

23. Trimarchi S, Eagle KA, Nienaber CA, et al. Role of age in acute type A aortic dissection outcome: report from the International Registry of Acute Aortic Dissection (IRAD). J Thorac Cardiovasc Surg 2010;140:784-9.

24. Biousse V, D'Anglejan-Chatillon J, Massiou H, et al. Head pain in non-traumatic carotid artery dissection: a series of 65 patients. Cephalalgia 1994;14:33-6.

25. Erbel R, Aboyans V, Boileau C, et al. [2014 ESC Guidelines on the diagnosis and treatment of aortic diseases].[Article in Polish]. Kardiol Pol 2014;72:1169-252.

26. Mokashi SA, Svensson LG. Guidelines for the management of thoracic aortic disease in 2017. Gen Thorac Cardiovasc Surg 2019;67:59-65.

27. Alter SM, Eskin B, Allegra JR. Diagnosis of aortic dissection in emergency department patients is rare. West J Emerg Med 2015;16:629-31.

28. Hiratzka LF, Bakris GL, Beckman JA, et al. 2010 ACCF/AHA/AATS/ACR/ASA/SCA/SCAI/SIR/STS/SVM Guidelines for the diagnosis and management of patients with thoracic aortic disease: a report of the American College of Cardiology Foundation/American Heart Association Task Force on Practice Guidelines, American Association for Thoracic Surgery, American College of Radiology, American Stroke Association, Society of Cardiovascular Anesthesiologists, Society for Cardiovascular Angiography and Interventions, Society of Interventional Radiology, Society of Thoracic 
Surgeons, and Society for Vascular Medicine. Circulation 2010;121:e266-369.

29. Kurabayashi M, Miwa N, Ueshima D, et al. Factors leading to failure to diagnose acute aortic dissection in the emergency room. J Cardiol 2011;58:287-93.

30. Rosman HS, Patel S, Borzak S, et al. Quality of history taking in patients with aortic dissection. Chest 1998;114:793-5.

31. Klompas M. Does this patient have an acute thoracic aortic dissection? JAMA 2002;287:2262-72.

32. Czerny M, Schmidli J, Adler S, et al. Current options and recommendations for the treatment of thoracic aortic pathologies involving the aortic arch: an expert consensus document of the European Association for Cardio-Thoracic surgery (EACTS) and the European Society for Vascular Surgery (ESVS). Eur J Cardiothorac Surg 2019;55:133-62.

33. Nicosia A, Greco G, Felis S, et al. [Diagnostic accuracy of transesophageal echocardiography in the diagnosis of aortic dissection: comparison with computerized axial tomography].[Article in Italian]. Cardiologia 1995;40:329-9.

34. von Tengg-Kobligk HM, Giesel FL. Contrast agents in radiology. In: Miller CK, Schwartz, LH, editors. Medical imaging in clinical trials. London: Springer; 2014. p. 327-57.

35. Fukui T. Management of acute aortic dissection and thoracic aortic rupture. J Intensive Care 2018;6:5. 